\title{
Agôn
}

Revue des arts de la scène

$5 \mid 2012$

L'entrée en scène

\section{Eros et Thanatos}

Entrées en scène du corps dans le cinéma de Steve McQueen

Alice Leroy

\section{(2) OpenEdition}

Journals

Édition électronique

URL : http://journals.openedition.org/agon/2337

DOI : 10.4000/agon.2337

ISSN : 1961-8581

Éditeur

Association Agôn

Référence électronique

Alice Leroy, «Eros et Thanatos », Agôn [En ligne], 5 | 2012, mis en ligne le 14 janvier 2013, consulté le

19 avril 2019. URL : http://journals.openedition.org/agon/2337 ; DOI : 10.4000/agon.2337

Ce document a été généré automatiquement le 19 avril 2019.

Association Agôn et les auteurs des articles 


\title{
Eros et Thanatos
}

\author{
Entrées en scène du corps dans le cinéma de Steve McQueen
}

Alice Leroy

Michael Fassbender, Hunger, de Steve McQueen, 2008

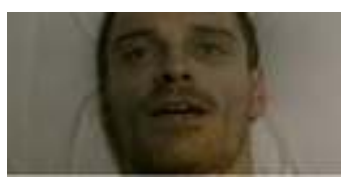

Michael Fassbender, Shame, de Steve McQueen, 2011

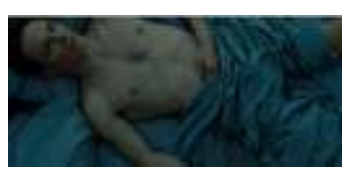

1 Dans le long plan fixe qui inaugure Shame (2011), second long-métrage de fiction du cinéaste britannique Steve McQueen, Brandon (Michael Fassbender) est allongé nu dans un lit aux draps défaits, la main posée sur le bas-ventre, le regard perdu dans le vague, la caméra en plongée restant longtemps sur son corps immobile. La fixité de son regard seul un clignement d'œil nous convainc au bout d'un certain temps qu'il est bien vivant rappelle l'un des tout derniers plans de Hunger (2008), celui où Bobby Sands (le même Michael Fassbender), amaigri et apaisé, visage blanc et regard vide, repose sur son lit de mort. Étrange contiguïté de ces deux plans en plongée sur des corps alités, l'un et l'autre figés dans une espèce d'absence à soi, à la fois présents dans l'espace du cadre et comme hypnotisés par un point invisible par-delà la caméra, fixant un hors champ auquel nous, spectateurs, n'avons pas accès. Sans doute ce hors champ est-il celui de la mort pour Bobby Sands, arrivé au terme de sa grève de la faim; peut être est-il pour Brandon celui de la culpabilité, celle qui donne son titre au film et l'introduit comme une figure de gisant dans ce premier plan baigné d'une lumière crue de petit matin, le réveil sonnant inlassablement. Au-delà de l'évidente différence entre ces deux corps, l'un bien vivant et l'autre agonisant, leur station couchée et leur immobilité tendent à les abstraire de l'espace du cadre, comme s'ils refusaient tous deux de passer à l'action, de « jouer le jeu » 
du récit. Ce refus de présence au cadre a valeur matricielle dans le cinéma de Steve McQueen, qui a lui-même inauguré ce dispositif dans un court-métrage, Illuminer (2001) ${ }^{1}$. Le cinéaste y apparaît en personne, étendu nu et immobile sur un lit, seulement éclairé par le halo de lumière bleutée de la télévision. La passivité de son corps, ombre sombre allongée au milieu des draps défaits, contraste avec le débit rapide d'un reporter qui commente une mission de l'armée française en Afghanistan. Cette frontalité de l'image d'un corps opposant sa passivité à l'action (militaire, que la voix du téléviseur hors champ commente) d'une part, et au regard du spectateur d'autre part, accuse l'un des paradoxes essentiels du cinéma selon Steve McQueen : celui de l'incarnation. Dans la frontalité toute théâtrale de cette caméra en plongée au-dessus de corps alités, le cinéaste dénonce cette corporéité qui fait précisément défaut au cinéma, art d'images animées par le truchement de la projection. L'entrée en scène y est une opération mécanique, alors qu'elle est physique au théâtre. Et Steve McQueen de montrer qu'un personnage peut être présent au cadre tout en refusant de s'y inscrire, en se soustrayant, par son indifférence et sa passivité, au regard de la caméra. Il nous faut dès lors considérer cette qualité propre au théâtre et qui semble manquer au cinéma : comment ce médium parvient-il à faire entrer $\mathrm{du}$ corps dans l'image? Comment nous fait-il éprouver le corps qu'il représente? Comment lui donne-t-il consistance et chair ? L'entrée en scène dans le cinéma de Steve McQueen ne relève pas seulement d'une forme d'introduction des personnages dans l'espace du cadre (mise en scène) ou dans celui du récit (scénario), elle détermine plutôt un régime d'incarnation: entrer en scène dans Hunger ou dans Shame, c'est parvenir à entrer en corps.

2 On a pu reprocher à Steve McQueen d'être plus plasticien que cinéaste et de délaisser par exemple le contre-champ historique des grèves de la faim de 1981 en Irlande du Nord au profit d'une esthétisation de l'enfermement et de l'agonie dans Hunger. Cette critique se trouve néanmoins infondée dès lors qu'on ne considère plus les films de Steve McQueen comme de vivants tableaux mais comme la reformulation politique et esthétique de la question de l'incarnation au cinéma: dans Hunger comme dans Shame, le récit s'inscrit dans les matières d'images (durées, fixité et mouvement, montage) et dans l'épreuve des corps, si bien que l'entrée en scène des personnages est avant tout celle de corps qui portent la charge narrative du film. Le corps y est d'emblée présenté comme le lieu du combat politique (Hunger) ou bien de la damnation (Shame). Envisageant cette problématique de l'incarnation dans le cinéma de Steve McQueen sous l'angle de l'entrée en scène des corps, nous entendons montrer d'une part que celle-ci ne coïncide nullement avec le commencement du film, et qu'elle relève d'autre part d'une épreuve physique qui appartient tout à la fois au registre de la performance et à celui de l'action politique.

\section{Entrée en scène, commencements et recommencements}

3 Il n'est pas indifférent qu'en dépit de leurs dissemblances irréductibles, les deux longs métrages de fiction tournés par Steve McQueen avec l'acteur Michael Fassbender en 2008 et 2011 inscrivent le corps dans un espace panoptique : de façon littérale pour Hunger, qui évoque les grèves de la faim dans les Blocks $\mathrm{H}$ de la prison de Long Kesh en Irlande du Nord en 1981 avec la figure emblématique de Bobby Sands ; à travers une métaphore plus économique dans Shame, sur les pas d'un cadre supérieur new yorkais accroc au sexe au 
point d'inonder son ordinateur de vidéos pornographiques et de recourir aux services de prostituées à la moindre pause-déjeuner. Le jeu des transparences, des gratte-ciels de Manhattan ou des écrans scintillants d'ordinateur, des parois de verre cloisonnant l'espace de travail de Brandon ou des vitrines à travers lesquelles il observe une femme désirée, déterminent un régime de sur-visibilité auquel nul ne peut échapper. Brandon le sait bien, lui qui vit dans la honte de voir un jour ses déviances exposées à la vindicte publique. C'est dès lors moins son personnage proprement dit qui entre en scène que l'espace même de la ville dans l'incipit du film, imposant un rythme monotone et répétitif à l'existence de Brandon: succession de matins gris, mornes trajets de métro jusqu'au bureau ponctués par le souvenir de coïts sans passion. La séquence d'ouverture de Shame organise minutieusement ce rituel qu'on sait déjà invariable: la récurrence des gestes quotidiens (ouvrir le store, écouter le répondeur, prendre un verre d'eau) et sons usuels (la voix de Sissy sur le répondeur, l'eau de la douche) scande le quotidien de Brandon. Arrivent ensuite les proies, femmes croisées dans le métro ou dans un bar, qui éveillent à nouveau ses instincts et rouvrent ce cycle infernal de consommation rapide et de honte dont il est prisonnier. De sorte que si Bobby Sands apparaît au terme de sa longue agonie comme un martyr ayant enfin trouvé le repos, Brandon évoque dès le plan inaugural de Shame, un éternel damné, subissant son calvaire à chaque jour recommencé, à l'image d'un Prométhée sans cesse dévoré par ses propres pulsions. Obsédé par son désir jamais assouvi, Brandon a des airs de sociopathe qui l'inscrivent dans la parenté du Patrick Bateman de Bret Easton Ellis ${ }^{2}$. Mais ses pulsions ne vont jamais jusqu'au meurtre, et McQueen n'en propose aucun motif valable. Brandon n'est ni un prédateur qui trouverait dans une sexualité débridée une échappatoire à une vie d'ennui, ni un séducteur invétéré (il laisse volontiers la place à son patron, d'ailleurs piètre dragueur). À bien y réfléchir, il se situe même à l'opposé de ces rôles et n'aspire qu'à devenir aussi transparent que le monde de surfaces translucides et d'écrans qui l'entoure. Il parle peu, reste étonnamment passif dans les situations de rencontres qui précèdent le passage à l'acte et semble privilégier les relations tarifées avec des prostituées pour aller directement à l'essentiel. Il fait preuve d'une indolence qui le rapproche moins du charismatique Patrick Bateman campé par Christian Bale chez Mary Harron (American Psycho, adapté du roman de Bret Easton Ellis en 2000) que de la distante Claire Dolan, le personnage éponyme du deuxième film de Lodge Kerrigan (1998). Claire (Katrin Cartlidge), une call-girl à Manhattan - de celles-là mêmes à qui Brandon fait souvent appel - cherche en vain à échapper à la ronde quotidienne de ses " passes " de bureaux. Comme dans Shame, les façades de verre et chambres d'hôtels standards de Manhattan y sont le décor d'un commerce sexuel de routine. La démesure verticale de l'architecture urbaine et l'échelle de l'homme face à ces tours hirsutes accusent les différences entre gloires futiles et destins misérables, dans une ville où la réussite est, au moins en apparence, une question d'étage (la vision d'un clochard endormi dans le métro au cours de la première séquence de Shame en est une vivante image). Le visage impassible de Katrin Cartlidge ou de Michael Fassbender, l'étalonnage froid des scènes de sexe et le pragmatisme de ces étreintes de bureau, épargnent aux deux films l'écueil du mélodrame et n'appellent aucune compassion. Leur sexualité quasi-mécanique est à la fois en deçà et au-delà de tout moralisme.

4 Hunger repose sur la même dynamique de la répétition et du recommencement : comme dans Shame, le film ne commence pas avec la séquence d'ouverture, mais recommence un rituel infiniment rejoué, celui de l'univers carcéral. Tandis que l'enfermement n'est esquissé que sous l'égide de la métaphore urbaine dans Shame, il est ici littéral avec une 
architecture pénitentiaire dans laquelle la caméra embarque le spectateur. La séquence d'ouverture de Hunger suit ainsi les pas d'un bourreau ordinaire, le gardien de prison Raymond Lohan, et ceux de Davey Gillen, jeune détenu qui découvre la « blanket and nowash protest $\|^{3}$ en actes. De la même manière que Shame met d'abord en scène l'espace urbain, ce long prologue offre une vision édifiante de l'institution carcérale. Motif récurrent du cinéma (au point d'en constituer d'ailleurs un genre à part entière), la prison impose ses propres codes de représentation au cinéma et McQueen en adopte ici la grammaire : avec la figure du surveillant taciturne, le ballet des grilles, les couloirs vides et silencieux, et surtout la figure du novice qui entre en prison comme on entre en religion, au terme d'un rituel codifié de dépossession de ses droits et de ses biens. Entrer en prison - et le nouveau détenu l'apprendra rapidement - c'est se trouver réduit à l'espace du corps, dernier bastion de résistance. Néanmoins, Steve McQueen se garde bien de montrer l'exercice de la violence sur les corps durant ce prologue, préférant laisser le spectateur avancer dans le film comme s'il avait « les yeux bandés ", de manière à ce que "progressivement par le toucher, il appréhende les choses; avec l'arrivée de Bobby, le bandeau tombe et il se réveille $»^{4}$. L'on entre dans le quartier de haute sécurité de Long Kesh sur les traces de Raymond Lohan le maton, et avec le grand corps hébété de Gillen se laissant guider par la main menottée à son bras. On n'en sortira pas, sinon pour assister fugacement à l'exécution de Lohan, un bouquet de marguerites à la main, s'effondrant sur les genoux de sa mère cataleptique à l'hospice, une autre institution " totale » au sens de Goffman ${ }^{5}$. C'est donc une entrée en espace plutôt qu'une entrée dans la temporalité du récit qu'organisent les prologues de Shame et d'Hunger.

5 Ce n'est pas avant la vingt-cinquième minute du film que s'ouvre le temps de la narration, avec l'entrée en scène du personnage principal, Bobby Sands, leader charismatique des Blocks $\mathrm{H}$ de Long Kesh. Longtemps différée par les ellipses de la double séquence d'ouverture - les hématomes sur la tête de Gillen, les poings tuméfiées de Lohan sont autant d'indices de la régularité des coups dans cet univers - la violence surgit en même temps que le personnage de Sands. C'est un sinistre rituel d'ablutions forcées qui en fournit le prétexte : « Get ready! ( (« Prépare-toi ! ») prévient le co-détenu du jeune Gillen. Arrachés à leurs cellules, les prisonniers sont roués de coups et plongés dans un bain d'eau glacé pour y être sommairement frottés à coups de balai brosse avant d'être ramenés inconscients et presque noyés dans leurs geôles. Dans ce déchaînement de coups et de cris, apparaitt pour la première fois le corps nu et hirsute de Bobby Sands, hurlant et se débattant entre les bras armés de ses tortionnaires. Rasé grossièrement à grands coups de ciseaux, le visage tuméfié, il lutte comme un diable pendant que Lohan et deux autres gardiens le maintiennent fermement sous l'eau. Vaincu, il est porté inconscient dans sa cellule, la tête couverte d'hématomes dodelinant doucement en contre-plongée sous la lumière blanche du couloir. Tenu à bout de bras par les gardiens et filmé en contreplongée, il offre déjà l'image christique qui l'identifiera comme un martyr quand il entamera une grève de la faim.

6 Il est patent que l'entrée en scène ne concerne jamais que les personnages masculins, tandis que les personnages féminins se trouvent relégués dans une espèce de hors-scène qui ne se confond pas toujours avec un hors-champ: Sissy, la sœur de Brandon, est bien présente au cadre et néanmoins jamais elle ne parvient à entrer en scène dans la vie de son frère ou d'un autre homme. Incidemment chez Steve McQueen, les voix féminines sont souvent désincarnées : à l'instar de Sissy qui n'est, dans la première séquence de Shame, qu'un lointain écho sur le répondeur ou de Margaret Thatcher dans Hunger dont la 
voix (archive radiophonique et seul élément de réalité que Steve McQueen utilise dans sa mise en scène $e^{6}$ ) plane tel un fantôme sur les couloirs vides du Maze. Le corps féminin n'entre en scène qu'à titre d'intrus : Sissy accomplit en effet une double effraction d'une part en pénétrant l'intimité de son frère - son appartement d'un blanc virginal et d'une propreté impeccable - et d'autre part en apparaissant nue sous la douche alors qu'il croit surprendre un cambrioleur, puisqu'elle offre sa nudité au regard de son frère comme le seul corps féminin dont il ne peut supporter la vision. Le corps de Sissy ne peut pas exister dans l'économie libidinale de Brandon et il est par conséquent inévitable que cette négation de son corps en même temps que de sa présence incongrue dans la vie de son frère la conduise au suicide. En miroir du corps libidinal de son frère, Sissy est un noncorps, une voix désincarnée sur une messagerie téléphonique - ce qu'elle redevient dans l'ultime séquence du film une fois que Brandon l'a abandonnée dans l'appartement en lui signifiant de quitter les lieux avant son retour. Le suicide de Sissy et l'agonie de Sands ont ceci de commun qu'ils forment deux tentatives distinctes pour retrouver une voix. Pulsion de mort et pulsion de vie opposent ainsi deux extrêmes du corps, qui culminent dans les dernières séquences de Hunger et Shame: la puissance de l'autodestruction à travers la déchéance du corps dans le premier, l'énergie libidinale au bord du désespoir d'une longue nuit d'errance et d'étreintes dans le second. Loin d'incarner deux régimes antagonistes, l'Eros et le Thanatos recherchent les mêmes états limites, produisent tous deux une transe. De sorte que l'extase libidinale de Brandon ne peut sans doute coïncider qu'avec l'agonie de Sissy, qui se tranche les veines en l'absence de son frère.

\section{Mise à l'épreuve de soi et logique d'épuisement du corps}

7 Dans le cinéma de Steve McQueen, le commencement s'avère un éternel recommencement et ne coïncide jamais avec l'entrée en scène des personnages. On pourrait aisément récuser toute parenté entre le détenu républicain gréviste de la faim du Maze (le «labyrinthe») et le trader dépendant sexuel de Manhattan, ce serait néanmoins ne considérer ces corps que comme des motifs narratifs dans l'œuvre du cinéaste. Or Steve McQueen s'attache à monter des corps arrimés à un système de représentation dont ils sont prisonniers (tout entier symbolisé par l'espace dans lequel ils s'inscrivent, la mégalopole ou la prison). L'entrée en scène du corps, retardée (Hunger) ou bien euphémisée (Shame), se joue donc sur un terrain politique aussi bien qu'esthétique : il s'agit toujours pour un personnage d'échapper aux cadres de représentation que lui imposent son environnement, de manière à pouvoir incarner son propre corps.

8 Néanmoins, "l'Histoire avec sa grande hache » selon l'expression de Perec, bien qu'elle compose de toute évidence l'arrière-plan d'Hunger, n'entre pas en scène dans le cinéma de Steve McQueen et l'archive filmique n'y est jamais convoqué. Aucun flashback ne vient non plus raviver les souvenirs de Brandon et Sissy dans Shame: le passé peut bien peser comme un fardeau entre eux, il n'est jamais évoqué qu'à mots couverts et le frère comme la sœur semblent le regarder comme l'origine du mal qui les ronge ${ }^{7}$. En dépit de tout ce qui les distingue sur le plan dramaturgique, Hunger et Shame ont en commun de réduire le corps à sa seule condition organique et de le soumettre à des situations limites. Non pas celles qu'instaurent la discipline carcérale ou la rationalité économique appliquée aux rapports sociaux et sexuels et qui contraignent les détenus du Maze dans le dénuement et Brandon dans l'opprobre, mais celles auxquelles se soumettent eux-mêmes les 
personnages. L'un et l'autre film sont ainsi construits selon un rythme d'intensité, un souffle de l'ordre de l'effort physique. En vertu de cette prégnance du corps sur le rythme de la narration, Steve McQueen s'intéresse peu à la réalité historique ou à la biographie de ses personnages. Hunger n'use d'aucun subterfuge narratif pour raconter la vie passée des détenus et les motifs de leur incarcération ${ }^{8}$. S'il avait voulu montrer l'injustice de ces conditions d'arrestation, Steve McQueen eut pourtant trouvé dans la biographie de Bobby Sands matière à raconter le parcours tragique et typique d'un gosse de Belfast. Dédaignant la vérité historique ou la vraisemblance de son intrigue, Steve McQueen conçoit ses œuvres comme des épreuves d'endurance, pareilles à la course d'orientation du jeune Bobby Sands dont celui-ci se souvient tandis qu'il agonise sur son lit en prison ; aussi n'est-on pas surpris de retrouver dans Shame un long travelling nocturne sur la course de Brandon le long d'avenues new yorkaises dont il étouffe le bruit avec les Variations Goldberg de Bach dans ses écouteurs. Dans les deux cas, la course a valeur de mise à l'épreuve symbolique du corps, un épuisement maîtrisé (la seule façon pour Brandon de contrôler sa colère ou son désir), semblable en cela à celui du gréviste de la faim, qui se laisse volontairement et douloureusement mourir. Le cinéaste a éprouvé cette logique de l'endurance et de la durée dans sa pratique de vidéaste et plasticien : «le cinéma expérimental m'a appris qu'on peut faire durer le plan», explique-t-il ${ }^{9}$. Emblématique de la modernité cinématographique, le plan séquence offre bien plus qu'une scène à l'acteur chez Steve McQueen. Ses durées exceptionnelles ont valeur de mise à l'épreuve et d'exténuation du corps : ainsi le long dialogue de Bobby Sands et du père Dominic Moran (Liam Cunningham) dans Hunger ou le travelling qui suit durant près de cinq minutes la course régulière de Brandon dans Shame. La frontalité du dispositif filmique et la longueur des plans tirent le cinéma de Steve McQueen vers le théâtre et la performance ${ }^{10}$.

L'entrée en scène brutale de Sands à la vingt-cinquième minute du film ancre le personnage dans un cycle de souffrance qui ira désormais crescendo. À travers la mise en tension du corps dans des équilibres de plus en plus précaires, Steve McQueen organise la montée en puissance du récit de la lutte des hommes-couvertures... jusqu'à l'engagement radical du corps dans la grève de la faim. Hunger se déploie ainsi comme un retable : trois tableaux, le dernier répondant au premier, dessinent les formes ultimes d'engagement du corps dans l'action politique, à travers l'affrontement, l'éthique et le sacrifice. Cette construction en triptyque organise la logique spéculaire du premier et du dernier tableau : à la brutalisation des corps enfermés dans l'enfer carcéral répond la violence retournée contre soi du gréviste de la faim dans une agonie sacrée. Si Steve McQueen s'inscrit dans l'héritage d'un cinéma expérimental qui s'est attaché à explorer les états transgressifs du corps - dans sa nudité crue de fluides et d'excréments, sa sexualité hétéro- ou homo-érotique (Kenneth Anger ${ }^{11}$, Stan Brakhage ${ }^{12}$, James Broughton ${ }^{13}$, Andy Warhol ${ }^{14}$ ) voire ses lieux de déchéances, notamment l'hôpital et la morgue dans Deus Ex et The Act of seeing with one's own Eyes de Stan Brakhage (1971) - il s'en distingue néanmoins à deux titres au moins. D'une part, le corps porte un enjeu narratif dans Hunger et Shame: l'image de sa déchéance n'a pas vocation à transgresser les tabous de sa représentation, comme autrefois les œuvres de Kenneth Anger ou Stan Brakhage. D'autre part, Hunger et Shame convoquent une tradition picturale occidentale de l'extase et de la déchéance. À travers des entrées en scène composées comme de puissants tableaux animés, ils invoquent une même iconographie religieuse du pêché et de la souffrance. La nudité du corps dans ces entrées en scène vaut bien entendu pour le dénuement du personnage - au sens littéral pour le prisonnier politique de Long Kesh dans Hunger, au sens figuré pour 
Brandon, prisonnier de ses propres pulsions et de sa culpabilité dans Shame - mais elle renvoie plus profondément à la peinture érotique et religieuse : dans le plan inaugural qui le découvre étendu dans son lit et comme absent à lui-même, Brandon incarne une improbable odalisque masculine, esclave de sa propre passion, tandis que la figure émaciée de Sands dans le premier et l'ultime tableaux de Hunger - premier et troisième temps de son entrée en scène - invoque la passion du Christ, son corps décharné porté par ses bourreaux jusqu'à la croix. Cette iconographie érotique et religieuse ne vaut pas seulement au titre de référence esthétique : à rebours de tout fétichisme, la chair y fait l'objet d'un regard clinique bien plus que d'un regard de désir ou de pitié.

10 Ce n'est pas le dolorisme ou la passion qui interpellent Steve McQueen dans les images transgressives du corps, mais sa performance. Sans doute faut-il y lire la filiation revendiquée du cinéaste avec le cinéma muet et ses dispositifs frontaux. Dans Deadpan, qui lui vaut le prix Turner en 1999, Steve McQueen réinvente par exemple la scène de Steamboat Bill Jr où Buster Keaton reste droit comme un I tandis que la façade d'une maison s'écroule sur lui et qu'il en sort miraculeusement indemne en passant au travers d'une fenêtre. Steve McQueen substitue son propre corps au corps burlesque de Keaton, « corps noir massif en lieu et place de l'acrobate chétif, impassible et statique lors des chutes répétées sous plusieurs angles de la façade qui vient finalement s'abattre sur le devant de l'image ", menaçant directement le spectateur, comme le remarque Antoine Thirion ${ }^{15}$. À l'intérieur du cadre se joue une succession de «chutes» de cet autre cadre qu'est la fenêtre, qui donne au corps sa verticalité dans un univers où tout s'effondre. Dans ce court-métrage, Steve McQueen élabore déjà une conception de l'entrée en scène où seul le corps détermine l'enjeu du récit: le corps de l'acteur - en l'occurrence, du cinéaste - résiste à l'effondrement du décor et l'entrée en scène ne coïncide pas avec le commencement du film qui n'est, pour ainsi dire, que la répétition d'un mouvement infini (la chute de la façade). Dans la continuité de cet héritage du muet, on pourrait considérer l'entrée en scène dans le cinéma de McQueen comme une prise de corps plutôt que comme une prise de parole, le personnage de Brandon dans Shame résumant en une formule lapidaire cette destitution du langage : «Actions count, not words $»^{16}$.

11 Les chairs couvertes d'hématomes et les murs tapissés d'excréments de Hunger avaient installé Steve McQueen dans le paysage cinématographique comme un cinéasteplasticien. Dans Shame, les murs de l'appartement de Brandon affichent désormais une blancheur virginale et Sissy dissimule ses cicatrices sous ses vêtements. Même enfoui sous la honte et affamé jusqu’à la désincarnation, le corps demeure pourtant indélébile. On aurait tort cependant de postuler que Steve McQueen cherche seulement la performance ou bien une quelconque esthétique de la transgression dans ces états-limites du corps. Entrer en scène ou prendre corps devient pour le cinéaste un enjeu politique tout autant qu'esthétique et narratif. Il s'agit d'inventer un registre d'incarnation qui prend forme au fur et à mesure des entrées en scène du corps dans l'espace du cadre, la temporalité du récit, mais aussi la picturalité du plan. Brandon ou Bobby Sands ne prennent chair qu'à mesure qu'ils délimitent les frontières de leur propre corps, qu'ils en déterminent la singularité à rebours des cadres de représentation dans lesquels ils se trouvaient contraints au début du film. La force iconographique tout autant que politique de ces apparitions détermine dès lors un régime d'incarnation inédit dans un cinéma où l'entrée en scène ne se confond pas avec l'incipit des films - celui-ci n'étant jamais que la répétition quasi-mécanique d'un destin toujours rejoué. Steve McQueen livre ainsi une 
étrange métaphore du cinéma, défilement automatique et toujours recommencé de photogrammes désincarnés qui laissent pourtant affleurer une présence à leur surface.

\section{NOTES}

1. Illuminer de Steve McQueen (Royaume-Uni, 2001, vidéo, couleur, 15 min 13 s).

2. Dans American Psycho.

3. Littéralement « la grève des couvertures et de l'hygiène », mouvement de protestation contre les conditions de détention engagé par les détenus des Blocks $\mathrm{H}$ à la fin des années 1970.

4. Ciment Michel, «Les raisons de vivre et les raisons de mourir », entretien avec Steve McQueen, Positif, $n^{\circ} 573$, novembre 2008. p. 14.

5. Goffman Erving, Asiles, Études sur la condition sociale des malades mentaux. Les Éditions de Minuit, coll. « Le sens commun », Paris : 1968, p. 41. Il définit l'institution totalitaire comme un « lieu de résidence et de travail où un grand nombre d'individus, placés dans la même situation, coupés du monde extérieur pour une période relativement longue, mènent ensemble une vie recluse dont les modalités et les règles de vie sont explicitement et minutieusement réglées ».

6. L'intégralité du film a été tournée en studio.

7. Tous deux n'en parlent qu'à de rares occasions et toujours en des termes vagues et elliptiques : à David, le patron de Brandon qui tente de la séduire et découvre les cicatrices sur son poignet, Sissy explique en souriant: «When I was a kid I was bored» («Quand j'étais enfant, je m'ennuyais ») ; Brandon se contente quant à lui d'un résumé liminaire de son enfance alors que sa collègue Marianne lui demande d'où il est : «I was born in Ireland and we moved here when I was a teen » («Je suis né en Irlande et nous avons emménagé ici quand j'étais adolescent »).

8. Le seul flash-back que concède McQueen se trouve à la toute fin du film et n'a pas de fonction narrative.

9. Michel Ciment, «Le mouvement et le rituel ». Entretien avec Steve McQueen, Positif, n610, décembre 2011, p. 18.

10. Le plasticien a d'ailleurs toujours travaillé avec des auteurs dramatiques pour mettre en scène ses longs-métrages de fiction: Enda Walsh dans Hunger, et Abi Morgan dans Shame. À travers une théâtralité revendiquée, Steve McQueen cherche cet état de performance propre à la mise en jeu du corps dans des situations limites. On comprend dès lors l'importance de sa collaboration avec Michael Fassbender, acteur physique s'il en est, toujours prêt à donner de sa personne.

11. Dès Fireworks en 1947.

12. Flesh of Morning (1956), Wedlock House (1959).

13. The Bed (1968), The Golden Positions (1971).

14. Couch (1964), Blow Job (1964), My Hustler (1965), Blue Movie (1969)...

15. Thirion Antoine, «L'homme-sables », Les Cahiers du cinéma, op. cit. p. 44.

16. «Les actions comptent, pas les mots». 


\section{RÉSUMÉS}

Cinéaste venu de la vidéo et des arts plastiques, Steve McQueen interroge la corporéité propre à ce médium précisément désincarné qu'est le cinéma. Ses deux longs-métrages de fiction, Hunger (2008) et Shame (2011), formulent la question de l'incarnation au cinéma à travers une mise en scène des corps qui seule détermine l'ordre du récit. Aussi l'entrée en scène du corps chez Steve McQueen ne coïncide-t-elle jamais avec un quelconque commencement du film - celui-ci étant plutôt un éternel recommencent à l'instar de l'installation vidéo - mais élabore un geste à la fois esthétique et politique, afférant au registre de la performance aussi bien qu'à celui de l'action politique.

INDEX

Mots-clés : cinéma, McQueen (Steve), corps

\section{AUTEUR}

\section{ALICE LEROY}

Alice Leroy est doctorante contractuelle en études cinématographiques à l'Université d'Aix-

Marseille et chercheur associée à la Bibliothèque Nationale de France. Ses recherches portent sur une archéologie du corps de cinéma à l'aune de la notion foucaldienne de corps utopique. Elle a publié des articles dans les revues Esprit et Critique et contribue régulièrement à la revue en ligne Critikat. 